\title{
A simple UV completion for Higgs and Higgs-dilaton infla- tion
}

\author{
Dmitry Gorbunov ${ }^{1,2, *}$ and Anna Tokareva ${ }^{1, * *}$ \\ ${ }^{1}$ Institute for Nuclear Research of Russian Academy of Sciences, 117312 Moscow, Russia \\ ${ }^{2}$ Moscow Institute of Physics and Technology, 141700 Dolgoprudny, Russia
}

\begin{abstract}
In this work, we present a UV completion for the Higgs and Higgsdilaton inflation which can be reached by adding only one extra $R^{2}$ term in the gravity sector. We show that, in this case, the strong coupling scale is lifted up to the Planck scale.
\end{abstract}

\section{Introduction}

By the reason of minimality, it is tempting to exploit the Higgs field as the inflaton in the early Universe. However, in order to provide with a successful inflationary stage, one needs to have a large non-minimal coupling between the Higgs field and gravity [1]. Such coupling leads to troubles with the tree level unitarity at relatively low energy scales, compared to the Planck mass. Although the inflation itself can be described in the formalism of the fielddependent cutoff [6], during the preheating stage, it tends that a number of particles with momenta exciding the unitarity cutoff scale are produced $[9,10]$. This means that the consistent description of the inflation and reheating requires a wider framework which is valid up to the Planck scale.

In this work, we study an extension of the Higgs inflation model with an $R^{2}$ term in the sector of gravity and show that the unitary cutoff scale in all sectors of the model is of order the Planck mass. Also, we extend this statement to the scale-invariant modification of the Higgs inflation - Higgs-dilaton model [24]. We show that the addition of the $R^{2}$ term provides with a valid perturbative UV completion for both Higgs and Higgs-dilaton inflation models that allows for obtaining robust predictions consistent with observations.

\section{Extension of the Higgs inflation with the $R^{2}$ term}

The presence of the $R^{2}$ term is natural from the point of view of the quantum gravity effects [12]. Let us start with the action of the Higgs-inflation augmented with the squared scalar curvature term,

$$
S_{0}=\int d^{4} x \sqrt{-g}\left(-\frac{M_{P}^{2}+\xi h^{2}}{2} R+\frac{\beta}{4} R^{2}+\frac{\left(\partial_{\mu} h\right)^{2}}{2}-\frac{\lambda}{4}\left(h^{2}-v^{2}\right)^{2}\right) .
$$

\footnotetext{
*e-mail: gorby@ms2.inr.ac.ru

**e-mail: tokareva@ms2.inr.ac.ru
} 
At $\beta=0$ eq. (1) describes the model of Higgs-inflation ( $h$ stands for the Higgs field in unitary gauge). Here $\xi$ has to take the value in the range $\sim 10^{3}-10^{4} \gg 1$, in order to provide with correct predictions for CMB. [1, 13]. However, this large coupling is known to spoil the perturbative unitarity of the model at the energy scale $M_{P} / \xi$ [3].

If $\beta \neq 0$ the action (1) is known to provide with an extra scalar degree of freedom (scalaron) in the gravitational sector [14]. The mass of this particle is given by

$$
m=\frac{M_{P}}{\sqrt{3 \beta}} .
$$

Introducing a Lagrange multiplier $L$ and auxiliary scalar $\mathcal{R}$ we obtain from (1),

$$
S=\int d^{4} x \sqrt{-g}\left(\frac{\left(\partial_{\mu} h\right)^{2}}{2}-\frac{\lambda}{4}\left(h^{2}-v^{2}\right)^{2}-\frac{M_{P}^{2}+\xi h^{2}}{2} \mathcal{R}+\frac{\beta}{4} \mathcal{R}^{2}-L \mathcal{R}+L R\right) .
$$

Then the field $\mathcal{R}$ can be integrated out,

$$
S=\int d^{4} x \sqrt{-g}\left(\frac{\left(\partial_{\mu} h\right)^{2}}{2}-\frac{\lambda}{4}\left(h^{2}-v^{2}\right)^{2}+L R-\frac{1}{\beta}\left(L+\frac{1}{2} \xi h^{2}+\frac{1}{2} M_{P}^{2}\right)^{2}\right),
$$

and the large coupling $\xi$ is now moved to the potential term. The actual coupling constant is $\xi^{2} / \beta$ instead of $\xi$ which can well be smaller than unity, provided

$$
\beta \gtrsim \frac{\xi^{2}}{4 \pi}
$$

Thus, we expect that the problem corresponding to the large value of $\xi$ can be solved in this way. Below we show that this is indeed the case.

\section{Higgs inflation with the $R^{2}$ term in the Einstein frame}

Let us perform the Weyl transformation to the Einstein frame

$$
g_{\mu \nu} \rightarrow \Omega^{2} g_{\mu \nu}, \quad \Omega^{2} \equiv \frac{2 L}{M_{P}^{2}},
$$

and replace $L$ with $\phi$ (dubbed scalaron) introduced as

$$
\phi \equiv M_{P} \sqrt{\frac{2}{3}} \log \Omega^{2} .
$$

In terms of $h, \phi$ and the rescaled metric, action (4) reads [15]

$$
S=\int d^{4} x \sqrt{-g}\left(-\frac{R}{12}+\frac{1}{2} e^{-2 \phi}(\partial h)^{2}+\frac{1}{2}(\partial \phi)^{2}-\frac{1}{4} e^{-4 \phi}\left(\lambda h^{4}+\frac{1}{36 \beta}\left(e^{2 \phi}-1-6 \xi h^{2}\right)^{2}\right)\right)
$$

Hereafter we use a convention $M_{P}=1 / \sqrt{6}$. We make the Higgs field canonical in a different variables,

$$
h=e^{\Phi} \text { th } H, \phi=e^{\Phi} / \cosh H,
$$

which leads to the lagrangian in the scalar sector of the theory,

$$
L=\frac{1}{2} \cosh ^{2} H(\partial \Phi)^{2}+\frac{1}{2}(\partial H)^{2}-\frac{1}{4}\left(\lambda \sinh ^{4} H+\frac{1}{36 \beta}\left(1-e^{-2 \Phi} \cosh ^{2} H-6 \xi \sinh ^{2} H\right)^{2}\right) .
$$

In this variables, the Higgs field couples to gauge $W$-bosons (and similarly to $Z$-bosons) as

$$
L_{\text {gauge }}=\frac{g^{2} h^{2}}{4} e^{-2 \phi} W_{\mu}^{+} W_{\mu}^{-}=\frac{g^{2}}{4} \sinh ^{2} H W_{\mu}^{+} W_{\mu}^{-} .
$$




\section{Unitarity cutoff scale}

Let us find the strong coupling scales in the model (1) in the Einstein frame. There are three sectors to be examined.

Gravity sector. Since in the Einstein frame the action for gravitons has the standard form, the scattering between the gravitons happen similarly to those coming from the EinsteinHilbert term. The unitarity is known to be broken at the energies of order the Planck mass.

Scalar sector. Here we use the model action in the form (7) is useful. The interaction between Higgs field $h$ and scalaron $\phi$ is originated from the kinetic term (the second term of lagrangian (7)) and the potential. From the series in the field, $\phi$ one finds, that all nonrenormalizable terms come with the coupling suppressed by the Planck scale. ( $\phi$ always appears as $\left.\phi /\left(\sqrt{6} M_{P}\right)\right)$. Thus, the kinetic term in (7) is healthy up to the Planck scale. The same is true for the potential term of the lagrangian (7), provided the inequality (5), even if $\xi \gg 1$. This behavior was also found in Ref. [15]. Therefore, we conclude that with model parameters obeying (5) the scalar sector is free from the strong coupling problem up to the Planck scale.

Gauge sector. In the SM, the self-interaction of gauge bosons produces a part of the $2 \rightarrow 2$ scattering amplitude which grows with the particle momenta above the electroweak scale, $\propto p^{2} / m_{W}^{2}$. However, this part coming from the scattering of the longitudinal modes is canceled by the vortices including the exchange of the Higgs boson, see Fig. 1.
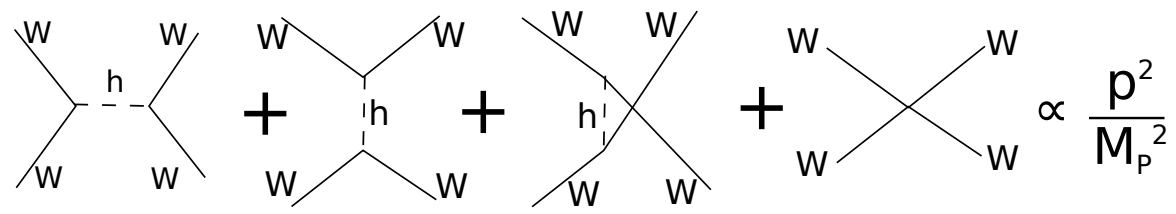

Figure 1. Scattering of electroweak massive gauge bosons.

If the Higgs sector is changed this compensation doesn't hold anymore. The growing part of the amplitude has the form,

$$
\mathcal{A} \sim \frac{g^{2} p^{2}}{m_{W}^{2}}\left(\frac{4}{g^{2}}\left(\frac{d m_{W}(H)}{d H}\right)^{2}-1\right) .
$$

In our model the canonically normalized Higgs field couples to the gauge bosons via the term (10). This leads to the mass term for the gauge bosons of the form $m_{W}=g \sinh H / 2$ and hence the amplitude is

$$
\mathcal{A} \propto \frac{p^{2}}{M_{P}^{2}} .
$$

Thus, the unitarity cutoff scale for the scattering of the gauge bosons is the Planck mass (here we restore the Planck mass, according to our convention $M_{P}=1 / \sqrt{6}$ ).

In the fermionic sector of the Standard model, the scattering amplitude of two fermions to two gauge bosons will grow linearly with the momentum (see [25]). Namely, the growing part is

$$
\mathcal{A}_{f} \sim y g \frac{p}{m_{W}}\left(\frac{2}{g}\left(\frac{d m_{W}(H)}{d H}\right)-1\right) \propto \frac{p}{M_{P}} \frac{\cosh H-1}{\sinh H} .
$$

Thus, the corresponding unitarity cutoff scale in the fermionic sector is always higher than the Planck mass. 


\section{Two-field inflation and predictions}

The model predictions for cosmological parameters are similar to those of the original Higgsinflation ${ }^{1}$ provided that the single field approximation is valid for this model [19]. The inflationary trajectory lies in a deep valley given by the equation (see Fig. 2) which corresponds
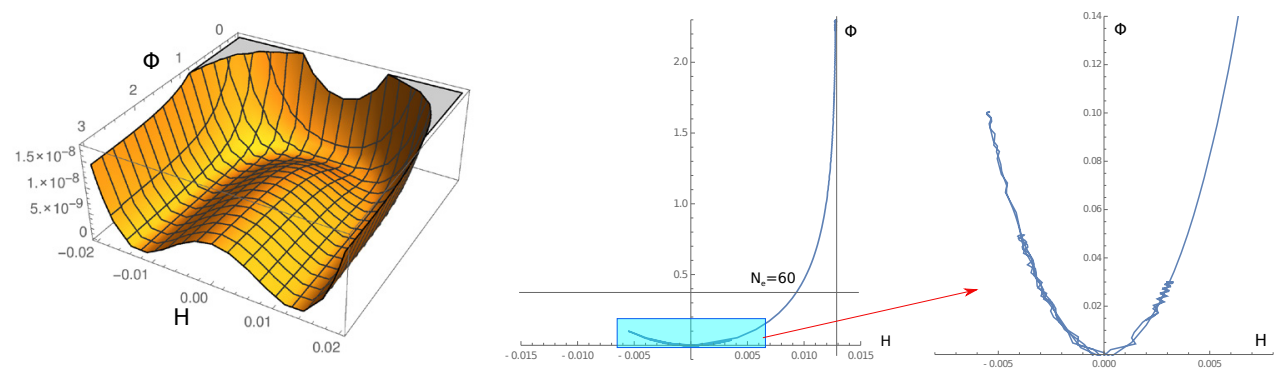

Figure 2. Potential and inflaton trajectory in the $R^{2}$ model which provides a UV completion for the Higgs inflation. At the beginning of inflation, the rolling degree of freedom does not interact with the SM particles. At the end of inflation, the trajectory turns leading to an efficient reheating due to the production of the SM gauge bosons. Notice that in [23] the potential looks somewhat different due to another choice of variables.

to the condition

$$
1-e^{-2 \Phi} \cosh ^{2} H-6 \xi \sinh ^{2} H=0,
$$

so that only the first term in potential (9) contributes to the energy density. In the general case, to obtain the amplitude of CMB fluctuations of order $10^{-5}$, one needs a normalization condition [15],

$$
\beta+\frac{\xi^{2}}{\lambda} \simeq 2 \times 10^{9}
$$

We can speak about the Higgs-scalaron inflation as a UV completion of the Higgs inflation if the CMB amplitude is actually defined by parameters of the Higgs sector, $\lambda$ and $\xi$, rather than $\beta$. The heavy degree of freedom indeed can be integrated out if $\beta<\xi^{2} / \lambda^{2}$. The mass of the heavy degree of freedom during inflation is of order $M_{P} / \sqrt{\xi}$ which is much higher than the Hubble scale. This means that deriving $\Phi$ through $H$ from the condition (14) and inserting it back to the action would provide with the effective action for the single field inflation which exactly matches with the original Higgs inflation model.

In this case, the predictions for the tilt of the scalar perturbation spectrum $n_{s}-1$ and tensor-to-scalar ratio $r$ are of the standard form ${ }^{3}$ [1],

$$
n_{s}=1-\frac{2}{N_{e}}, \quad r=\frac{12}{N_{e}^{2}}
$$

with $N_{e}=50 \div 60$ being a number of e-foldings of inflation which depends slightly on the reheating temperature. These predictions fall right in the ballpark of the region allowed by the Planck experiment [2]. No significant isocurvature and non-gaussianity are expected since the mass of the orthogonal direction is significantly larger than the Hubble scale (see Fig. 3).

\footnotetext{
${ }^{1}$ Or $R^{2}$-inflation [16], the difference in predictions of the two models is minuscule, for details see Refs. [17, 18].

${ }^{2}$ Notice that larger values of $\beta$ (which correspond to the light scalaron) are not allowed by the normalization condition (15) if $\xi$ is fixed. However, if $\xi$ is small the value of $\beta$ is fixed since it defines the amplitude of the scalar perturbations.

${ }^{3}$ This fact allows to distinguish this model from other UV completions for Higgs inflation suggested in the literature [20-22]. These works consider an addition of the extra scalar field. The cosmological predictions in this case typically depend on the parameters of this hidden scalar.
} 


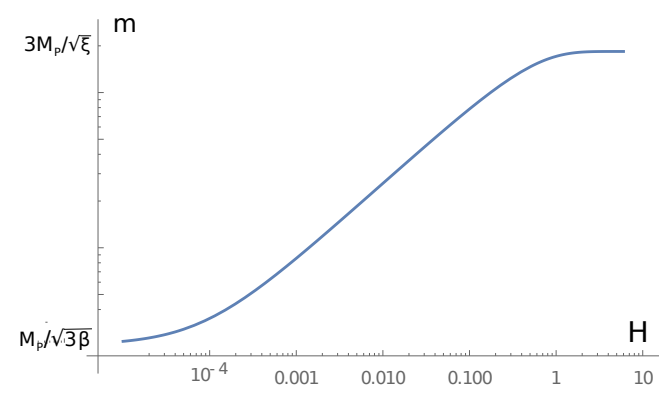

Figure 3. The dependence of the effective mass of the isocurvature mode (orthogonal to the inflaton trajectory) on the value of field $H$. Notice that during inflation this mass is of order $M_{P} / \sqrt{\xi}$ while at smaller $H$ it becomes $M_{P} / \sqrt{3 \beta} \lesssim M_{P} / \xi$. This behaviour is similar to the field-dependent cutoff scale in the Higgs inflation [6], something that is expected since this heavy degree of freedom provides a UV completion.

Summarising the bound (15) and perturbativity condition (5) on the parameter $\beta$, we find that this parameter should be in the range

$$
\frac{\xi^{2}}{4 \pi}<\beta<\frac{\xi^{2}}{\lambda}
$$

Thus, with typical value of $\lambda \sim 0.01$ at large values of the Higgs field the remaining window for the scalaron mass (2) is about three orders of magnitude. Consequently, for the reference value $\lambda=10^{-2}$, the scalaron mass is in the interval $5 \times 10^{13} \mathrm{GeV}<m<1.5 \times 10^{15} \mathrm{GeV}$.

\section{Completion for the Higgs-dilaton inflation}

In this section, we show that the $R^{2}$ term can cure the strong coupling problem not only in the Higgs inflation. A possible scale invariant extension of this model known as Higgs-dilaton inflation [24] also suffers from the similar problem with the low cutoff scale. This model yields the Planck mass and naturally small Higgs mass from the spontaneous breaking of the scale symmetry. Under certain choice of parameters, it provides with a viable inflationary stage. However, in this model, the Higgs field has to be coupled to gravity with large $\xi$ which again leads to the strong coupling scale about $M_{P} / \xi$, the same as in the original Higgs inflation.

The action of the Higgs-dilaton model completed with the $R^{2}$ term reads,

$$
S=\int d^{4} x \sqrt{-g}\left[\frac{1}{2}\left[\beta R^{2}+\left(\partial_{\mu} X\right)^{2}-\xi X^{2} R-\xi^{\prime} h^{2} R+\left(\partial_{\mu} h\right)^{2}\right]-\frac{\lambda}{4}\left(h^{2}-\alpha^{2} X^{2}\right)^{2}\right]
$$

In the Einstein frame this action can be written in such variables that the Higgs field direction becomes canonically normalized (see also Ref. [26] for a different choice of field variables where the scalaron field is canonical),

$$
\begin{aligned}
L & =\frac{1}{2}\left((\partial H)^{2}+\cosh ^{2} H(\partial \varphi)^{2}+\cosh ^{2} H \cosh ^{2} \varphi(\partial \rho)^{2}\right)- \\
& -\frac{1}{4}\left(\lambda\left(\sinh ^{2} H-\alpha^{2} \sinh ^{2} \varphi \cosh ^{2} H\right)^{2}+\frac{1}{36 \beta}\left(1-6 \xi \sinh ^{2} \varphi \cosh ^{2} H-6 \xi^{\prime} \sinh ^{2} H\right)^{2}\right) .
\end{aligned}
$$


Here field $\rho$ plays a role of the Goldstone boson of the broken scale invariance. It does not contribute to the potential. The field $\varphi$ corresponds to the scalar degree of freedom coming from gravity. The field $H$ is the only field coupled to the gauge and fermion sectors of the SM with the interaction term exactly of the form (10). Consequently, there is no strong-coupling issue in the gauge sector of the model, as well as in the scalar-gravity sector. The cutoff scale of such model is again pushed up to the Planck scale.
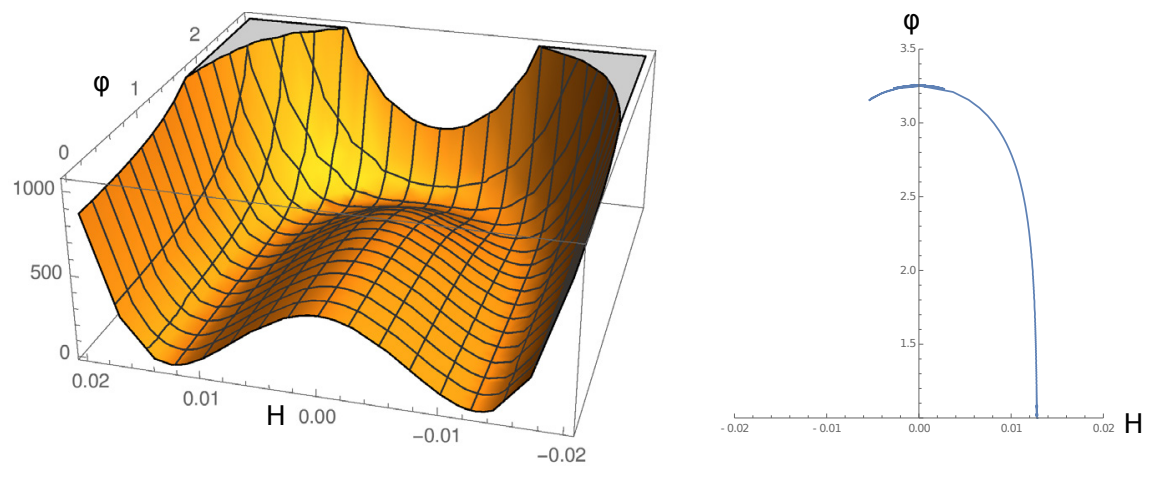

Figure 4. Potential and inflaton trajectory in Higgs-dilaton inflation with $R^{2}$ term.

The inflaton potential looks similar to the $R^{2}$-Higgs case. Again, the inflationary stage can be effectively described as the single field rolling inside the valley, under the conditions (17) on $\beta$, see Fig. 4. The predictions for spectral parameters are the same as in the Higgs-dilaton model [24]: the scalar tilt depends on the value of $\xi$,

$$
n_{s}=1-8 \xi \operatorname{coth} 4 \xi N_{e} .
$$

Therefore, in order to satisfy Planck limits [2], we need $\xi \lesssim 0.004$. The CMB amplitude can be obtained under the same condition as in (15).

\section{Improving the stability of the Higgs potential}

Finally, as an extra bonus, the introduced $R^{2}$ term can improve the stability of the Higgs potential. The latter is known to take negative values at large fields if the central value of the top quark mass is considered, for details see [27]. The top Yukawa coupling contributes to the renormalization group running of the Higgs self-coupling $\lambda$, such that, given the central value of the top quark mass, it is hard to reach the positive energy density during inflation (see [28]). The scalaron provides a positive one-loop contribution to the beta-function of $\lambda$ [12],

$$
\delta \beta_{\lambda}=\frac{1}{16 \pi^{2}} \frac{2 \xi^{2}(1+6 \xi)^{2}}{9 \beta^{2}} .
$$

Thus, in presence of the $R^{2}$-term the stability of the Higgs potential can be preserved for larger values of the top quark mass $m_{t}$ (see Fig. 5).

Notice also that if $\lambda$ is negative in some region of large fields, the Higgs field would stay in the false vacuum during reheating. However, due to the large reheating temperature, the thermal corrections could finally bring the Higgs to the SM vacuum [29]. While the detailed study of this process in our model is required, we expect that the domain of the top quark masses consistent with viable inflation becomes wider than in the minimal Higgs inflation. 

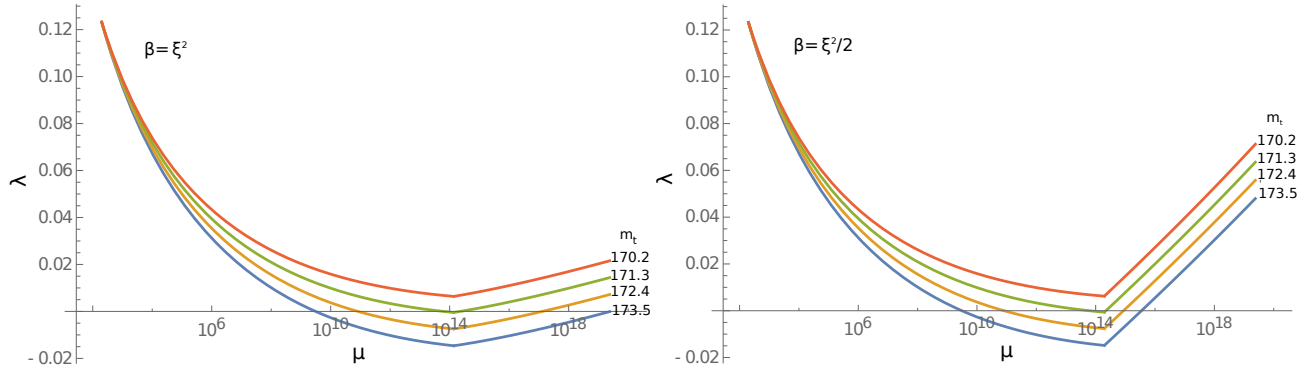

Figure 5. The dependence of the Higgs self-coupling $\lambda$. Here we plot the running of the parameter $\lambda$ which stands in the potential (7). Due to the matching condition [15], this is exactly the paramer describing the low energy Higgs scattering. on the renormalization scale $\mu$ with the scalaron one-loop impact included. The latter affects the running starting from the scale of order the scalaron mass (2).

\section{Conclusions}

In this work, we found that the addition of the extra gravitational scalar through the $R^{2}$ term yields to the rising of the unitarity cutoff scale to the Planck mass for inflationary models with large non-minimal couplings, such as Higgs inflation and Higgs-dilaton inflation. Thus, such a model can be treated as a UV completion valid for the energies lower than the Planck mass. This is a good improvement for Higgs and Higgs-dilaton models which suffer from the unitarity breaking problem, especially during the preheating stage. A completion with the $R^{2}$ term can be considered as a minimal extension of the original model since there is only one extra free parameter which also appears to be bounded (17). This separates the $R^{2}$ case from the models suggested earlier which extend the SM with a hidden scalar sector with a number of free parameters. Thus, the predictions for the scalar perturbations appear to be robust and independent of the parameter $\beta$ (scalaron mass) if (17) is satisfied.

The first thing that is expected to depend on $\beta$ is the reheating process. Since the particles with momenta higher than the cutoff scale of the original model are produced, the details of reheating would depend on the UV completion, i. e. on the parameter $\beta$ of the considered model. We leave this issue for a future study. Another thing that would be defined by the concrete value of $\beta$ is a form of the potential with quantum corrections included. However, we expect the significant corrections if the running of the Higgs self-coupling brings $\lambda$ to the domain of negative values, see Fig. 5 or if the scalaron mass is too large. In other cases, the classical description provides with valid results, given the value of $\lambda$ is taken at the appropriate energy scale.

The authors are grateful to F. Bezrukov, M. Shaposhnikov, S. Sibiryakov for valuable discussions. This work was supported by Russian Science Foundation grant 14-22-00161.

\section{References}

[1] F. L. Bezrukov and M. Shaposhnikov, Phys. Lett. B 659, 703 (2008)

[2] P. A. R. Ade et al. [Planck Collaboration], Astron. Astrophys. 594, A20 (2016)

[3] C. P. Burgess, H. M. Lee and M. Trott, JHEP 0909 (2009) 103

[4] J. L. F. Barbon and J. R. Espinosa, Phys. Rev. D 79 (2009) 081302

[5] F. Bezrukov, D. Gorbunov and M. Shaposhnikov, JCAP 1110 (2011) 001

[6] F. Bezrukov, A. Magnin, M. Shaposhnikov and S. Sibiryakov, JHEP 1101 (2011) 016

[7] F. Bezrukov, D. Gorbunov and M. Shaposhnikov, JCAP 0906, 029 (2009) 
[8] J. Garcia-Bellido, D. G. Figueroa and J. Rubio, Phys. Rev. D 79 (2009) 063531

[9] M. P. DeCross, D. I. Kaiser, A. Prabhu, C. Prescod-Weinstein and E. I. Sfakianakis, Phys. Rev. D 97 (2018) no.2, 023528

[10] Y. Ema, R. Jinno, K. Mukaida and K. Nakayama, JCAP 1702 (2017) no.02, 045

[11] F. Bezrukov, Class. Quant. Grav. 30 (2013) 214001

[12] I. G. Avramidi, "Covariant methods for the calculation of the effective action in quantum field theory and investigation of higher derivative quantum gravity,"

[13] F. Bezrukov and M. Shaposhnikov, JHEP 0907 (2009) 089

[14] K. S. Stelle, Gen. Rel. Grav. 9, 353 (1978).

[15] Y. Ema, Phys. Lett. B 770 (2017) 403

[16] A. A. Starobinsky, Phys. Lett. B 91 (1980) 99; A. A. Starobinsky, "Nonsingular model of the Universe with the quantum-gravitational de Sitter stage and its observational consequences," in: Proc. of the Second Seminar "Quantum Theory of Gravity" (Moscow, 13-15 Oct. 1981), INR Press, Moscow, 1982, pp. 58-72 (reprinted in: Quantum Gravity, eds. M.A. Markov, P.C. West, Plenum Publ. Co., New York, 1984, pp. 103-128).

[17] F. L. Bezrukov and D. S. Gorbunov, Phys. Lett. B 713 (2012) 365

[18] D. Gorbunov and A. Tokareva, JCAP 1312 (2013) 021

[19] Y. C. Wang and T. Wang, Phys. Rev. D 96, no. 12, 123506 (2017)

[20] G. F. Giudice and H. M. Lee, Phys. Lett. B 694, 294 (2011)

[21] H. M. Lee, Phys. Lett. B 722, 198 (2013)

[22] H. M. Lee,

[23] M. He, A. A. Starobinsky and J. Yokoyama, JCAP 1805, no. 05, 064 (2018)

[24] J. Garcia-Bellido, J. Rubio, M. Shaposhnikov and D. Zenhausern, Phys. Rev. D 84, 123504 (2011)

[25] F. Bezrukov, G. K. Karananas, J. Rubio and M. Shaposhnikov, Phys. Rev. D 87, no. 9, 096001 (2013)

[26] D. Gorbunov and A. Tokareva, Phys. Lett. B 739, 50 (2014)

[27] A. V. Bednyakov, B. A. Kniehl, A. F. Pikelner and O. L. Veretin, Phys. Rev. Lett. 115 (2015) no.20, 201802

[28] F. Bezrukov, M. Pauly and J. Rubio, JCAP 1802, no. 02, 040 (2018)

[29] F. Bezrukov, J. Rubio and M. Shaposhnikov, Phys. Rev. D 92, no. 8, 083512 (2015) 